\title{
The chemical enrichment history of the Magellanic Clouds field populations
}

\author{
Ricardo Carrera ${ }^{1}$, Carme Gallart ${ }^{2}$, Antonio Aparicio ${ }^{2}$, \\ Edgardo Costa $^{3}$, Eduardo Hardy ${ }^{3,4}$, Rene A. Méndez ${ }^{3}$, \\ Noelia E. D. Noël ${ }^{2}$ and Robert Zinn ${ }^{5}$ \\ ${ }^{1}$ Osservatorio Astronomico di Bologna, Via Ranzani 1, I-40127 Bologna, Italy \\ email: ricardo.carrera@oabo.inaf.it \\ ${ }^{2}$ Instituto de Astrofísica de Canarias, Vía Láctea sn, E-38200 La Laguna, Spain \\ ${ }^{3}$ Departamento de Astronomía, Universidad de Chile, Casilla 36-D, Santiago, Chile \\ ${ }^{4}$ National Radio Astronomy Observatory, Casilla El Golf 16-10, Las Condes, Santiago, Chile \\ ${ }^{5}$ Department of Astronomy, Yale University, New Haven, USA
}

\begin{abstract}
We report the results of our project devoted to study the chemical enrichment history of the field population in the Magellanic Clouds using Ca II triplet spectroscopy.
\end{abstract}

Keywords. galaxies: abundances, Magellanic Clouds, galaxies: stellar content

\section{Introduction}

The study of the ages and metallicities of the resolved stars in a nearby galaxy provides very detailed information on its evolutionary history. The Magellanic Clouds are examples of galaxies where this method can be applied very successfully. However, their vastness and our limitations in observing sizable samples of stars imply that there are particularly large gaps of knowledge in this area, compared with others. For example, their chemical enrichment histories have mainly been studied from their cluster systems. However, these objects have some drawbacks, like the age-gap of the LMC clusters or the single old cluster known in the SMC. Although the SMC is more metal-poor than the LMC, the chemical evolution of the cluster systems has been qualitatively similar in both galaxies. They show a first episode of chemical enrichment followed by a period of slow chemical evolution until around 4 Gyrs ago. Then, both galaxies took off again (Olszewski et al. 1991; Piatti et al. 2001). However, as mentioned above, there are some epochs in which the lack of clusters makes it difficult to extract definite conclusions. The chemical evolution of the Magellanic Clouds has also been investigated from their planetary nebulae (Dopita et al. 1991; Idiart et al. 2007), which show a behaviour similar to the clusters one. Finally, Cole et al. (2005) have studied the chemical evolution of the LMC bar based on a sample of red giant branch (RGB) stars. The age - metallicity relationship (AMR) of the bar stars is similar to the cluster's one, particularly for the older ages. However, the increase of metallicity observed in the clusters in the last 3 Gyrs is not observed in the bar.

What about the field populations in the LMC disk and in the SMC? Do they share the cluster and planetary nebulae behaviour in each galaxy? Does chemical evolution show a global pattern in each galaxy or on the contrary, does it depend on the position? To address these questions, and as part of a large project devoted to study the stellar content of the Magellanic Clouds, we have obtained metallicities and ages for stars in 4 large LMC and 13 SMC fields. In this paper we will discuss the procedure used to derive 
metallicities and ages, and describe our main conclusions on the chemical enrichment history of both Magellanic Clouds.

\section{Deriving stellar metallicities and ages}

Although the best way to derive chemical abundances is high-resolution spectroscopy, this technique needs huge amounts of telescope time to measure a significant number of stars even in the nearest galaxies. The alternative is low-resolution spectroscopy which, together with the modern multi-object spectrographs, allows us to observe large samples in a reasonable time. However, in external galaxies, even low-resolution spectroscopy is only possible for the brightest objects, which in most cases are RGB stars. A powerful index to derive metallicities in these stars is the infrared Ca II triplet (CaT). This index is linearly correlated with metallicity in the range $-2.2 \leqslant[\mathrm{Fe} / \mathrm{H}] \leqslant+0.47$, with no measurable influence of age in the interval $13 \leqslant$ Age/Gyr $\leqslant 0.25$ (Carrera et al. 2007). Tough this index may overestimate metallicities for values below -2.5 dex (Koch et al. 2008), the range of ages and metallicities in which it shows a linear behaviour agrees with the ones expected in the Magellanic Clouds.

The position of the RGB on the color - magnitude diagram (CMD) suffers from agemetallicity degeneracy. However, when the metallicity has been obtained in an alternative way, as in this case from spectroscopy, this age - metallicity degeneracy can be broken up, and stellar ages can be estimated from the position of the stars in the CMD. To do that, we computed a polynomial relationship to derive stellar ages from metallicities and positions of stars in the CMD. For that purpose, a synthetic CMD computed with IACSTAR (Aparicio \& Gallart 2004) with the overshooting BaSTI stellar stellar evolution models (Pietrinferni et al. 2004) as input was used. Details of this procedure can be found in Carrera et al. (2008a) and Carrera et al. (2008b).

\section{LMC chemical enrichment history}

In the LMC, we have studied four fields located at 2.3, 4.0, 5.5 and 7.1 northward of the bar. The CMDs of these fields have been presented by Gallart et al. (2008). We are obtaining detailed SFHs for them (see Gallart et al. 2008, and Meschin et al. in this volume) using the synthetic CMD technique. In each field we observed spectroscopically more than 100 stars in the upper part of the RGB. The results of this work can be found in Carrera et al. (2008a). Table 1 summarizes the mean metallicities and their dispersions in each field. The inner fields have a similar mean metallicity, while it is a factor of two more metal-poor in the outermost field.

Table 1. Mean values of metallicity distributions of LMC fields.

\begin{tabular}{lcc}
\hline Field & $\langle[\mathrm{Fe} / \mathrm{H}]\rangle$ & $\sigma_{[\mathrm{Fe} / \mathrm{H}]}$ \\
\hline Bar & -0.39 & 0.19 \\
2.3 & -0.47 & 0.31 \\
4.0 & -0.50 & 0.37 \\
$5^{\circ} .5$ & -0.45 & 0.31 \\
$7^{\circ} .1$ & -0.79 & 0.44 \\
\hline
\end{tabular}

To understand why the outermost field is more metal-poor, we obtained the AMR of each field (Fig. 1). The age error in each age interval is indicated in the top panel. The age distributions for each field have been plotted in inset panels taking into account (solid line) and not (histogram) the age determination uncertainties (see Carrera et al. 2008a for details). 


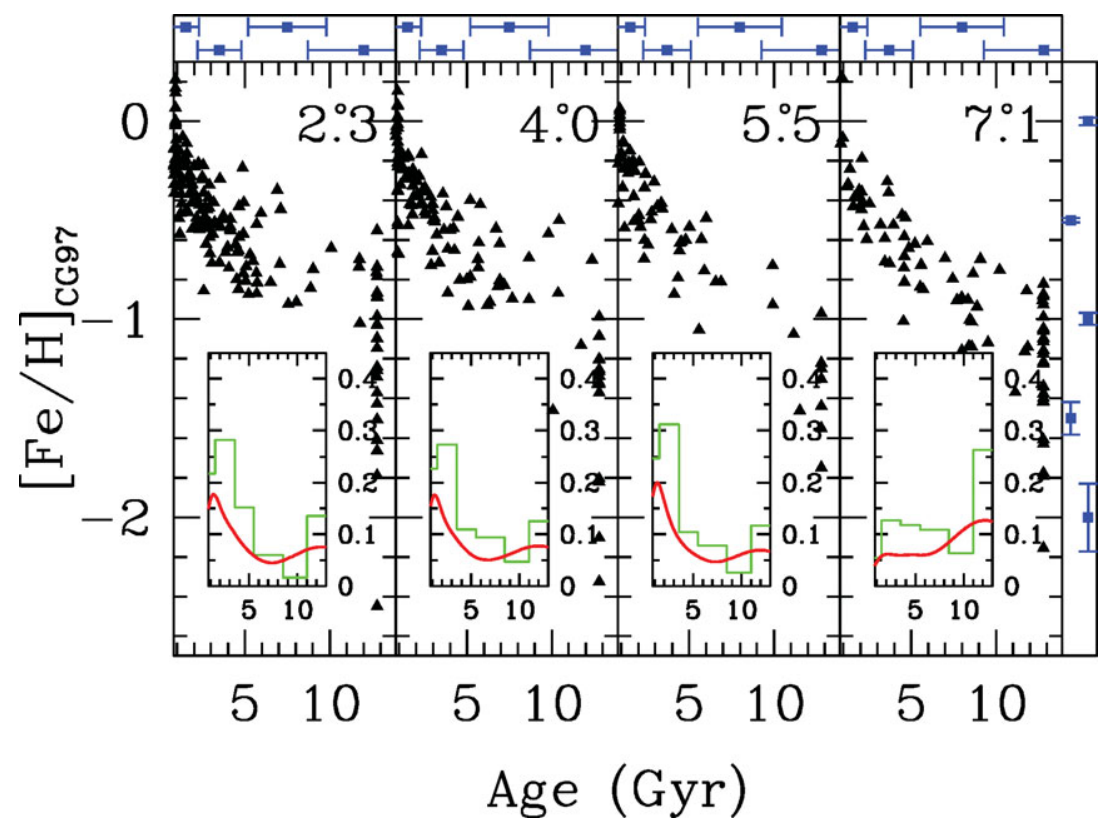

Figure 1. Age-metallicity relationships for the four LMC fields in our sample. Inset panels show the age distribution computed taking into account (solid line) and not (histogram) the age determination uncertainties. The top panel show the age error in each interval. The left panels show the metallicity error.

The AMR is, within the uncertainties, very similar for all fields, and also similar to the cluster ones. As expected, the most metal-poor stars in each field are also the oldest ones. A rapid chemical enrichment at a very early epoch is followed by a period of very slow metallicity evolution until around 3 Gyr ago, when the galaxy started another period of chemical enrichment that is still ongoing. Furthermore, the age histograms for the three innermost fields are similar, although the total number of stars decreases when moving away from the centre. The outermost field has a lower fraction of "young" (1-4 Gyr) intermediate-age stars. This indicates that its lower mean metallicity is related to the lower fraction of intermediate-age, more metal-rich stars rather than to a different chemical enrichment history (for example, a slower metal enrichment).

\section{SMC chemical enrichment history}

In the SMC we secured spectroscopy of stars in 13 fields spread about the galaxy body. The corresponding photometry has been published by Noël et al. (2007) and their detailed SFHs are presented in this volume by Noël et al. (2008). The positions of these fields, together with their mean metallicities are listed in Table 2. The fields are ordered by their distance to the center, which is shown in column 4 . Fields in different regions are indicated by different font types: eastern fields in normal, western fields in boldface and southern fields in italics. Mean metallicities are very close to $[\mathrm{Fe} / \mathrm{H}] \sim-1$ in all fields within $\mathrm{r} \lesssim 2$. 5 from the SMC center. A similar value is observed for the southern fields up to $\mathrm{r} \lesssim 3^{\circ}$ (qj0047 and smc0049). For the outermost fields, qj0033 in the West, and qj0102 and qj0053 in the South, the mean value is clearly more metal-poor than in the others. 
Table 2. SMC observed fields, mean metallicities and dispersion.

\begin{tabular}{lccccccc}
\hline Field & $\alpha_{2000}$ & $\delta_{2000}$ & $\mathrm{r}\left({ }^{\prime}\right)$ & $\mathrm{PA}\left(^{\circ}\right)$ & Zone & $\langle[\mathrm{Fe} / \mathrm{H}]\rangle$ & $\sigma_{[\mathrm{Fe} / \mathrm{H}]}$ \\
\hline smc0057 & $00: 57$ & $-73: 53$ & 65.7 & 164.4 & South & -1.01 & 0.33 \\
qj0037 & $\mathbf{0 0 : 3 7}$ & $\mathbf{- 7 2 : 1 8}$ & $\mathbf{7 8 . 5}$ & $\mathbf{2 9 4 . 0}$ & West & $\mathbf{- 0 . 9 5}$ & $\mathbf{0 . 1 7}$ \\
qj0036 & $\mathbf{0 0 : 3 6}$ & $\mathbf{- 7 2 : 2 5}$ & $\mathbf{7 9 . 8}$ & $\mathbf{2 8 8 . 0}$ & West & $\mathbf{- 0 . 9 8}$ & $\mathbf{0 . 2 5}$ \\
qj0111 & $01: 11$ & $-72: 49$ & 80.9 & 89.5 & East & -1.08 & 0.21 \\
qj0112 & $01: 12$ & $-72: 36$ & 87.4 & 81.0 & East & -1.16 & 0.32 \\
qj0035 & $\mathbf{0 0 : 3 5}$ & $\mathbf{- 7 2 : 0 1}$ & $\mathbf{9 5 . 5}$ & $\mathbf{3 0 0 . 6}$ & West & $\mathbf{- 1 . 0 9}$ & $\mathbf{0 . 2 4}$ \\
qj0116 & $01: 16$ & $-72: 59$ & 102.5 & 95.2 & East & -0.96 & 0.26 \\
smc0100 & $01: 00$ & $-74: 57$ & 130.4 & 167.5 & South & -1.07 & 0.28 \\
qj0047 & $00: 47$ & $-75: 30$ & 161.7 & 187.7 & South & -1.15 & 0.27 \\
qj0033 & $\mathbf{0 0 : 3 3}$ & $\mathbf{- 7 0 : 2 8}$ & $\mathbf{1 7 2 . 9}$ & $\mathbf{3 2 5 . 0}$ & West & $\mathbf{- 1 . 5 8}$ & $\mathbf{0 . 5 7}$ \\
smc0049 & $00: 49$ & $-75: 44$ & 174.8 & 184.6 & South & -1.00 & 0.28 \\
qj0102 & $01: 02$ & $-74: 46$ & 179.5 & 169.4 & South & -1.29 & 0.42 \\
smc0053 & $00: 53$ & $-76: 46$ & 236.3 & 179.4 & South & -1.64 & 0.50 \\
\hline
\end{tabular}

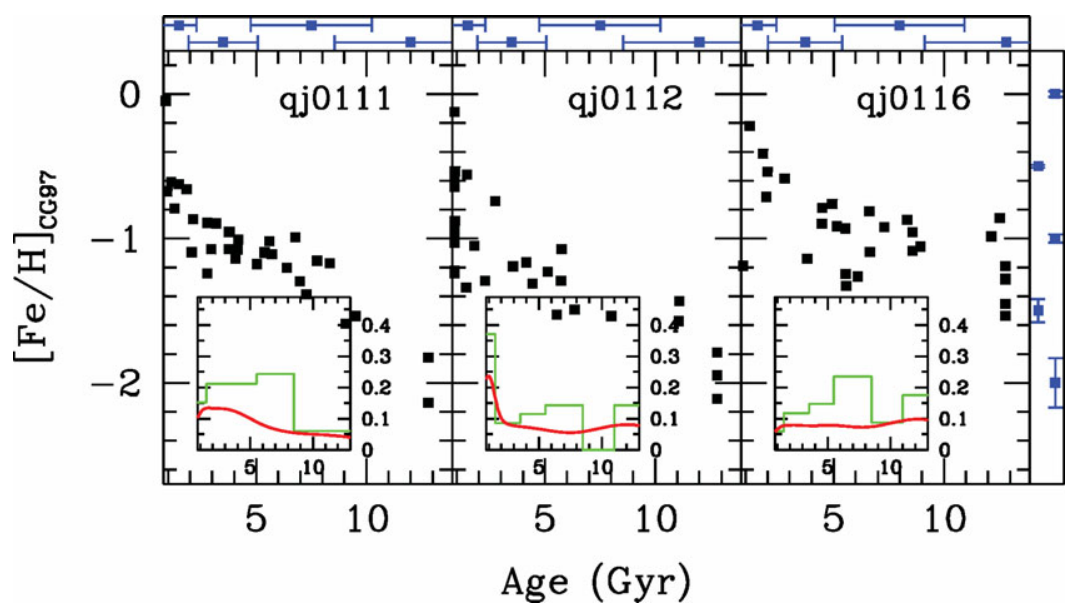

Figure 2. The same as Fig. 1, for the SMC eastern fields.

The fact that the mean metallicity decreases when moving away from the center implies that there is a metallicity gradient in the SMC. This is the first time that a spectroscopic metallicity gradient has been reported in SMC stellar populations. The detection of this gradient has been possible because we have covered a large radius range, up to $4^{\circ}$ from the SMC center.

To investigate the nature of the gradient, we have calculated the AMR for each field. They are plotted in Fig. 2, 3 and 4 for fields situated to the East, West and South respectively. Inset panels, as in Fig. 1, show the age distribution of the observed stars, with and without taking into account the age uncertainty (solid line and histogram, respectively).

All the AMR plotted in Fig. 2, 3 and 4 show a rapid chemical enrichment at a very early epoch. Even though in some fields we have not observed enough old stars to sample this part of the $\mathrm{AMR}$, note that $12 \mathrm{Gyr}$ ago all fields have reached $[\mathrm{Fe} / \mathrm{H}] \simeq-1.4$ to -1.0. This initial chemical enrichment was followed by a period of very slow metallicity evolution until around 3 Gyr ago. Then, the galaxy started another period of chemical enrichment, which is observed in the innermost fields, which are, however, the only ones where we observed enough young stars to sample this part of the AMR. In all cases, 


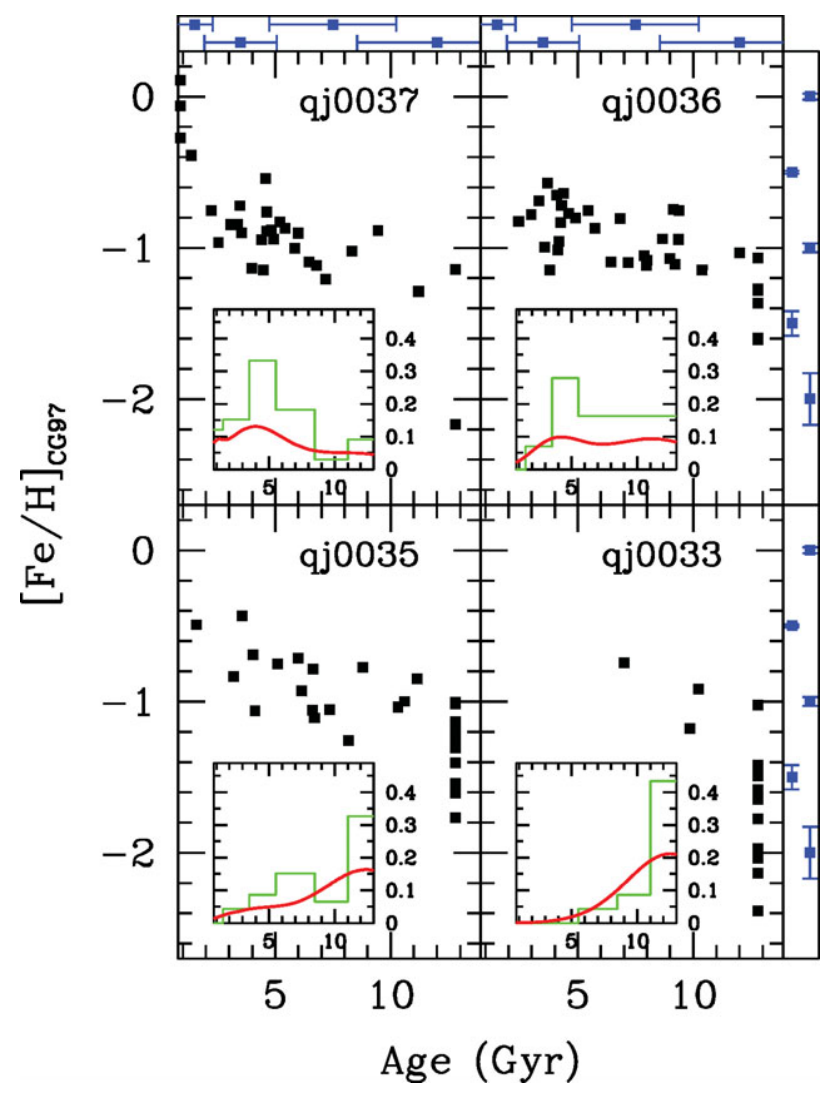

Figure 3. The same as Fig. 1, for the SMC western fields.

the mean metallicity is similar to that of the other fields at similar galactocentric radii. The field AMRs obtained in this work are similar to those for clusters (the reader should take into account that differences in the metallicity scales exist among different works), although there is only one cluster older than 10 Gyr (e.g., Piatti et al. 2001), and for planetary nebulae, with the exception that in these objects it is not observed the chemical enrichment episode at a very early epoch (Idiart et al. 2007).

For eastern fields, located in the wing, most of the observed stars have ages younger than 8 Gyr, but there is also a significant number of objects older than 10 Gyr. At a given galactocentric distance, eastern fields show a large number of young stars ( $\leqslant 3 \mathrm{Gyr}$ ) in comparison to the western ones. For the western and southern fields, the fraction of intermediate-age stars, which are also more metal-rich, decreases as we move away from the center, although the average metallicity in each age bin is similar. This indicates the presence of an age gradient in the galaxy, which may be the origin of the metallicity one. It is noticeable that for the most external fields, qj0033 and qj0053, we find a predominantly old and metal-poor stellar population.

\section{Future work}

We have investigated the chemical evolution of the field populations in both Magellanic Clouds. However, there are still some points which should be investigated. For example, in the LMC, we have studied 4 fields at different galactocentric distances northward of the bar. Now it is necessary to investigate what happens in other positions such as facing 


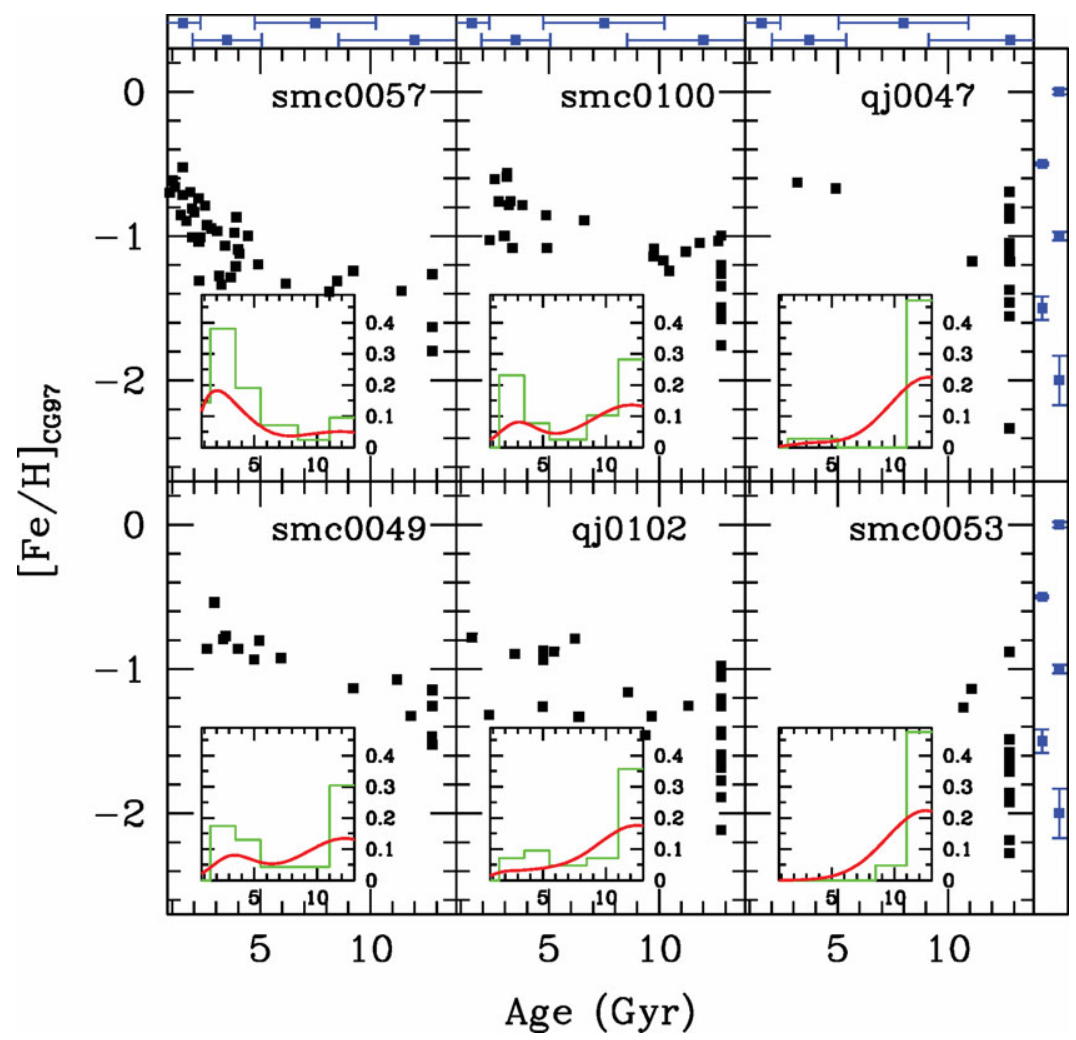

Figure 4. The same as Fig. 1, for the SMC southern fields.

the SMC and in the opposite direction. This could give us clues about the effects of the interactions with the SMC in the LMC field populations. Also, in both galaxies it is necessary to investigate the behaviour of the abundances of different chemical species as a function of position in the galaxy.

\section{References}

Aparicio, A. \& Gallart, C. 2004, AJ, 128, 1465

Carrera, R., Gallart, C., Pancino, E., \& Zinn, R. 2007, AJ, 134, 1298

Carrera, R., Gallart, C., Hardy, E., Aparicio, A., \& Zinn, R. 2008, AJ, 135, 836

Carrera, R., Gallart, C., Aparicio, A., Costa, E., Méndez, R. A., \& Nöel, N. E. D. 2008, AJ, 136,1039

Cole, A. A., Tolstoy, E., Gallagher III, J. S., \& Smecker-Hane, T. A. 2005, AJ, 129, 1465

Dopita, M. A., Vassiliadis, E., Wood, P. R., et al. 1997, ApJ, 474, 188

Gallart, C., Stetson, P. B., Meschin, I. P., Pont, F., \& Hardy, E. 2008, ApJ, 682, 89

Idiart, T. P., Maciel, W. J., \& Costa, R. D. D. 2007, A\&A, 472, 101

Koch, A., Grebel, E. K., Gilmore, G. F., et al. 2008, AJ, 135, 1580

Noël, N. E. D., Gallart, C., Costa, E., \& Méndez, R. A. 2007, AJ, 133, 2037

Olszewski, E. W., Schommer, R. A., Suntzeff, N. B., \& Harris, H. C. 1991, AJ, 101, 515

Piatti, A. E., Santos, J. F. C., Clariá, J. J., et al. 2001, MNRAS, 325, 792

Pietrinferni, A., Cassisi, S., Salaris, M., \& Castelli, F. 2004, ApJ, 612, 168 